\title{
III-Nitride Multi-Quantum-Well Light Emitting Structures with Selective Carrier Injection
}

\author{
Hussein S. El-Ghoroury, Mikhail V. Kisin * (1) and Chih-Li Chuang \\ Ostendo Technologies Inc., 6185 Paseo del Norte, Carlsbad, CA 92011, USA; hussein@ostendo.com (H.S.E.-G.); \\ chihli@ostendo.com (C.-L.C.) \\ * Correspondence: mikhail@ostendo.com
}

Received: 14 August 2019; Accepted: 12 September 2019; Published: 15 September 2019

check for updates

Featured Application: Multi-quantum-well light-emitting diodes, multi-color light emitters, white-light emitters.

\begin{abstract}
Incorporation into the multi-layered active region of a semiconductor light-emitting structure specially designed intermediate carrier blocking layers (IBLs) allows efficient control over the carrier injection distribution across the structure's active region to match the application-driven device injection characteristics. This approach has been successfully applied to control the color characteristics of monolithic multi-color light-emitting diodes (LEDs). We further exemplify the method's versatility by demonstrating the IBL design of III-nitride multiple-quantum-well (MQW) light-emitting diode with active quantum wells uniformly populated at LED operational current.
\end{abstract}

Keywords: III-nitride materials; carrier injection; multi-quantum-well structures; light emitting diodes

\section{Introduction}

Inhomogeneous carrier injection and an uneven population of optically active quantum wells unfavorably affects the performance of multiple-quantum-well (MQW) light emitters [1]. In III-nitride based LED structures, inferior hole transport is the commonly accepted source of inhomogeneous injection [2]. The excessive depth of InGaN quantum wells employed in visible-range light emitters and related increases in quantum well (QW) population capacity, however, are equally important causes of injection non-uniformity [3].

In light emitters based on other III-V materials (for instance, mid-infrared lasers), shallow and relatively wide quantum wells can be uniformly populated by efficient carrier exchange with highly mobile free-carrier subsystems [4]. Net confined charges of such quantum wells in LED operation mode are usually small. On the contrary, in deep-QW III-nitride heterostructures, the exchange between mobile and confined carriers is strongly biased toward the capture process. The latter is adversely affected by ballistic overshoot phenomena and becomes increasingly inefficient for narrow InGaN quantum well layers. As a result, the final established balance between the slow carrier capture and fast intra-QW recombination keeps the dynamic quantum well populations in profoundly away from equilibrium, and in case of different rates of electron and hole capture, also increases the net confined QW charge. In excessively deep green or red-emitting QWs, the deviation of confined carriers from an equilibrium with a mobile carrier subsystem becomes progressively stronger [5].

Since electrons and holes are injected from the opposite sides of the diode structure, their uneven distribution across the active region further upholds the inequality between quantum-confined electron-and-hole populations, which is especially noticeable in marginally located n-side and p-side quantum wells [6]. Residual confined charges, in turn, distort the active region's potential profile, and depending on the structure polarity, can either promote the quantum well ballistic overshoot [7] 
or enhance the carrier capture [8]. In c-plane polar III-nitride MQW LEDs, the resulting injection distribution usually favors the marginal p-side QW [9] which is amply populated with low-mobility holes, and therefore, always sustains enough net positive charge to attract mobile electrons for capture and recombination.

The mutual interdependence between QW populations and MQW injection distribution underlines the complexity of MQW device design and calls for application-dependent active region optimization. A strong interaction between different design elements makes MQW light emitter a nonlinear distributed system [1] so that all the active region constituents should be optimized simultaneously. In our approach, MQW injection rebalancing for a particular application is accomplished by including into the active region specially designed intermediate carrier blocking layers (IBLs) [10]; see Figure 1. The IBL design is comprised of both compositional and doping optimization and should be correlated with active quantum well compositions. The optimal IBL layout would also essentially depend on the required LED operational conditions like nominal bias voltage or injection current. This approach has been successfully applied to achieve the first full-color tunable monolithic LED covering complete RGB gamut [11,12], and recently, has been used to implement a white-color LED with tunable color temperature [13]. In this paper, we demonstrate yet another example of the IBL design of III-nitride MQW LED active region featuring a uniform population of active quantum wells at LED operational voltage.

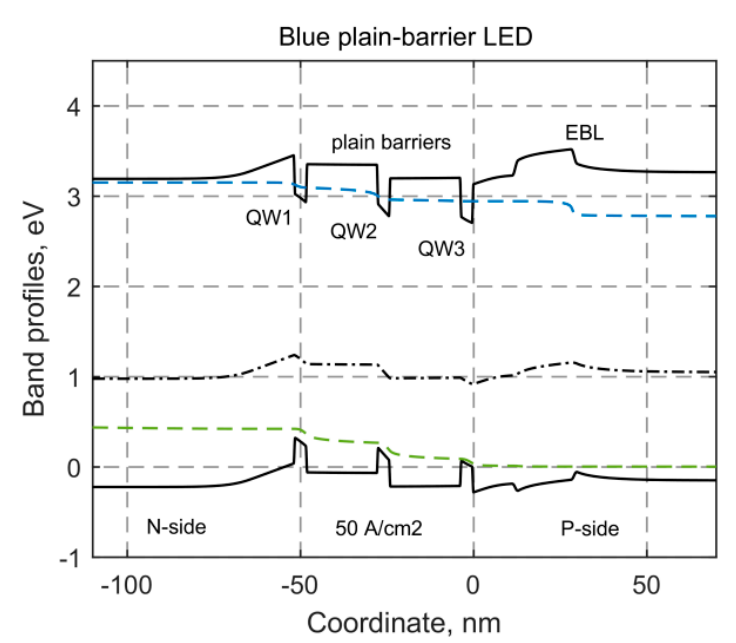

(a)

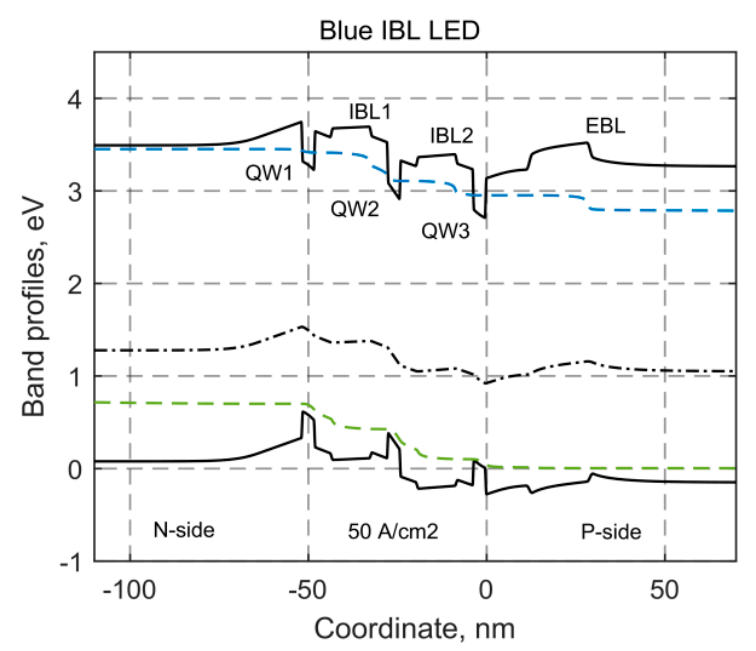

(b)

Figure 1. Band profiles of a blue-emitting LED with (a) a plain-barrier and (b) example intermediate carrier blocking layer (IBL) active region designs calculated at a nominal injection current of $50 \mathrm{~A} / \mathrm{cm}^{2}$. Dashed lines show the quasi-Fermi levels for electron (blue) and hole (green) drift-diffusion subsystems. The dashed-dotted line represents the internal built-in potential distribution across the active region.

\section{Methods}

For carrier transport simulation, the mobile electron and hole subsystems were further split into the drift-diffusion and high-energy parts, each assigned with different kinetic characteristics, such as carrier relaxation times, mobility, etc. [5]. The drift-diffusion carrier subsystems are described by equations of the standard drift-diffusion transport model complemented with rate equations for confined dynamic (non-equilibrium) QW populations, separately for each quantum well and for each type of carrier [6]. The high-energy electron subsystem includes Auger electrons supplied by intra-QW Auger recombination. High-energy electrons are assumed to fully relax into drift-diffusion electron subsystems within the span of the LED active region with characteristic relaxation time of 1 ps. Due to the much faster hole relaxation, the high-energy hole subsystem has not been included into the simulation. Low-energy drift-diffusion mobile carriers were assumed to be the only source of capture 
for QW-confined carriers; direct capture from the high-energy subsystem was ignored. Still, in the presence of barriers, high-energy Auger electrons can play a noticeable part in inter-QW transport, and therefore, affect MQW injection distribution [7,8]. Quasi-ballistic QW overshoot was incorporated into the drift-diffusion transport simulation by combining the QW capture characteristics with mobile low-energy carrier transit and relaxation times [14]. The final model equation system was solved by standard COMSOL Multiphysics ${ }^{\circledR}$ numerical solver without use of any specific application modules.

\section{Results}

The active region of monochromatic blue and green-emitting LEDs studied in this work includes three identical $4 \mathrm{~nm}$-wide GaInN QWs separated by $20 \mathrm{~nm}$ wide regions designed with or without the IBLs. In plain-barrier design, the regions separating the active QWs consist of GaN plain barriers only, while in IBL-designed structures, these regions comprise a $12 \mathrm{~nm}$ wide AlGaN IBL interposed between two adjacent QW barrier layers with $4 \mathrm{~nm}$ wide GaN buffers on each side. Figures 1 and 2 illustrate the active region layouts. In this example study, only $\mathrm{Al}$ compositions and doping levels of the IBLs have been varied, while the inter-QW separation was kept the same in all simulated structures to facilitate comparison between IBL and plain-barrier designs. LED layout also includes an external $\mathrm{p}^{+}$-doped $20 \mathrm{~nm} \mathrm{Al} \mathrm{fl}_{0.15} \mathrm{Ga}_{0.85} \mathrm{~N}$ electron blocking layer (EBL) located on the $\mathrm{p}$-side of the active region. Modeling shows, however, that the external EBL, while suppressing the electron leakage, can hardly affect the uniformity of MQW-carrier distribution across the active region at LED operational currents, and therefore, provides little impact on relative QW injection [3].

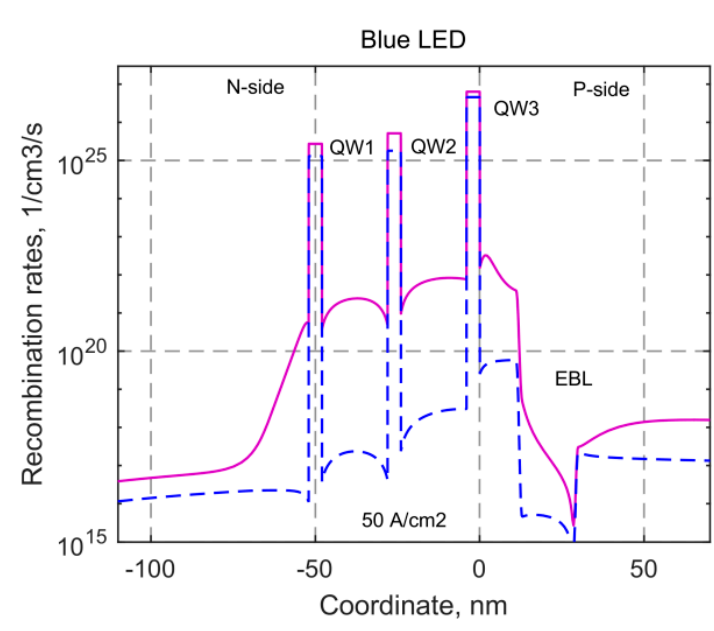

(a)

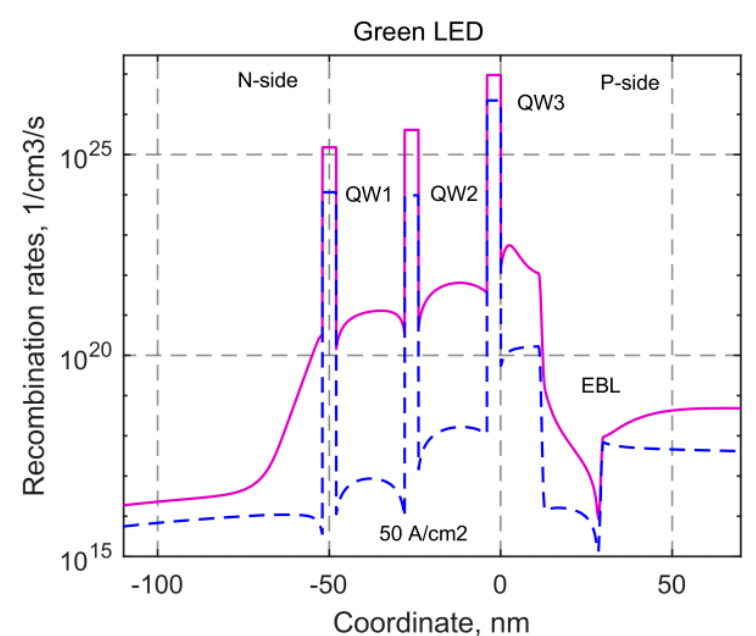

(b)

Figure 2. Recombination rate profiles across the active region of (a) blue-emitting and (b) green-emitting LEDs at a nominal injection current of $50 \mathrm{~A} / \mathrm{cm}^{2}$. Dashed lines indicate the radiative recombination rates.

QW radiative characteristics used in our example simulations are listed in Table 1. QW parameters have been calculated by independent QW simulation [5] assuming $40 \%$ values of interface polarization charges and using band structure characteristics taken from a single source [15]. We also adopted a conduction band-valence band offset ratio of $0.6 / 0.4$ established by the analysis of carrier leakage [16]. It should be noted that in a III-nitride material system, the characteristic parameter values have large variances, especially in the quantum wells. For our illustrative study, we tried to avoid the extremities of specific models and used parameter values which represent only the main features of the material system, which are the steady decrease of radiative and Shockley-Reed-Hall (SRH) recombination rate coefficients with increasing QW emission wavelength [17]. Parameter values in Table 1 represent this trend. The increase of SRH lifetimes in QWs with higher indium compositions corresponds to the higher level of compositional fluctuations which prevent the carriers from diffusing to SRH recombination centers $[18,19]$. Moderate variation of radiative recombination coefficients is 
due to efficient intra-QW polarization charge screening at high injection levels [20] paired with the possible balancing effect of compositional fluctuations in long-wavelength emitting QWs [21]. Auger coefficients for different QWs were assumed to be similar according to reference [17]. The relatively high values of QW Auger coefficients account for the enhancement of Auger processes in narrow QWs [22] which complies with the currently dominant view on the efficiency droop in III-nitrides as Auger-related phenomena [23,24]. The example values of the layer (2D) and bulk (3D) Auger coefficients are within the range of reported experimental findings [25-27]. The increase of capture times qualitatively reflects stronger carrier overshoot of QWs with higher polarization barriers [28].

Table 1. QW and bulk material parameters.

\begin{tabular}{cccc}
\hline QWs (Blue/Green/Red) & \multicolumn{3}{c}{ Bulk Material } \\
\hline Capture time, ps & $5 / 10 / 20$ & Mobility (electrons/holes), $\mathrm{cm}^{2} / \mathrm{V} \cdot \mathrm{s}$ & $200 / 10$ \\
SRH lifetime, ns & $20 / 40 / 60$ & SRH lifetime, $\mathrm{ns}$ & 1.0 \\
Auger coefficient, $10^{-16} \mathrm{~cm}^{4} / \mathrm{s}$ & $1.0 / 1.0 / 1.0$ & Auger coefficient, $10^{-30} \mathrm{~cm}^{6} / \mathrm{s}$ & 1.0 \\
Radiative constant $1,10^{-5} \mathrm{~cm}^{2} / \mathrm{s}$ & $3.0 / 1.5 / 1.0$ & Radiative constant, $10^{-10} \mathrm{~cm}^{3} / \mathrm{s}$ & 1.0 \\
QW emission, $\mathrm{nm}$ & $440 / 530 / 650$ & Energy relaxation time, $\mathrm{ps}$ & 1.0 \\
\hline
\end{tabular}

${ }^{1}$ For the nominal population $10^{13} \mathrm{~cm}^{-2}$.

Calculated band profiles of plain-barrier and IBL-designed LEDs simulated at the same with injection levels of $50 \mathrm{~A} / \mathrm{cm}^{2}$ are compared in Figure 1. The figure subplots also show the quasi-Fermi levels for mobile carriers controlled by drift-diffusion transport and the internal potential distribution inside the active region. Apparently, the IBL active region design, at the expense of slightly higher LED operational voltage of about $0.1 \mathrm{~V}$ per IBL, provides a more favorable potential profile around marginal n-side QWs. The extra height of the valence band profile around the n-side QW facilitates hole accumulation and ensures better hole supply for capture and subsequent intra-QW recombination. In plain-barrier active region LEDs, the n-side QWs are normally deprived of adequate hole injection, and, as a result, the p-side QW dominates the LED emission. Such injection misbalance is stronger in longer-wavelength emitting LEDs with deeper quantum wells. Figure 2 illustrates this trend by comparing the distributions of carrier recombination rates across the active regions of blue and green-emitting LEDs, with much higher injection and emission non-uniformity in the latter structure.

Figure 3 shows the results of IBL active region design optimization in which the IBL parameters have been chosen to alleviate the uneven MQW injection and uniformly populate the active QWs at a specific LED operational point. Figure 3 compares relative distributions of optical emission power among the active QWs $\left(P_{i} / \sum P_{i}\right)$ for blue-emitting and green-emitting designs of three-QW LEDs. Dashed lines present simulation results for plain-barrier LEDs without IBLs, while solid lines show corresponding distributions for LEDs with IBLs optimized for uniform injection at the usual LED operational current, around $50 \mathrm{~A} / \mathrm{cm}^{2}$. For optimal operation, the injection range should include the point of maximum LED external quantum efficiency (EQE). Simulated IBL-LEDs revealed a relatively uniform emission distribution in quite a wide injection range of practically important currents, $10-100 \mathrm{~A} / \mathrm{cm}^{2}$. At the same time, plain-barrier LEDs demonstrated the expected strong dominance of the marginal p-side QW, especially in the green-emitting LED structure. 


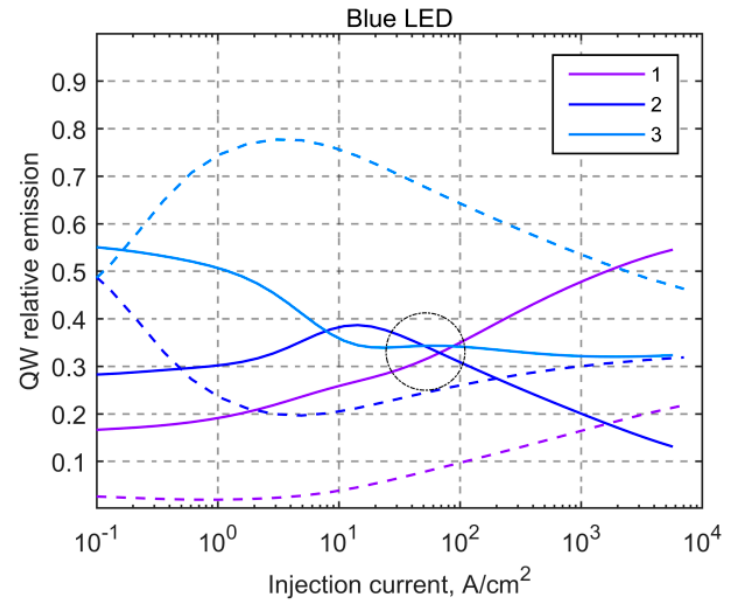

(a)

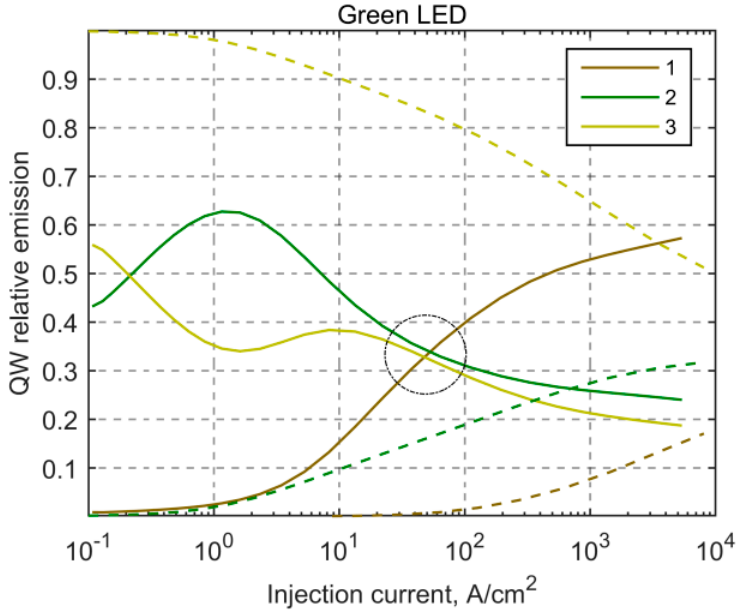

(b)

Figure 3. Injection dependence of multiple-quantum-well (MQW) relative optical emission in (a) blue-emitting (b) and green-emitting LEDs with plain-barrier (dashed lines) and optimized IBL (solid lines) active region designs. Quantum wells are counted from n-side of the active region. The area of the uniform MQW injection is encircled.

Figure 4 presents the injection dependence of MQW emission and injection uniformity for IBL LEDs. Uniformity characteristics are defined here as the ratios of optical emission powers $P_{i}$, and correspondingly, intra-QW radiative recombination currents $J_{i}$ for QWs with the lowest and highest levels of the relevant characteristics; e.g., minimum $\left(P_{i}\right)$ /maximum $\left(P_{i}\right)$. The optimization of LED operational conditions has not been specially considered in this work, though the peaks of calculated uniformity characteristics correlate quite well with the position of peak EQE for blue-emitting LED. Table 2 compares the emission uniformity characteristics of plain-barrier LEDs and IBL-LEDs at a nominal injection current of $50 \mathrm{~A} / \mathrm{cm}^{2}$ and shows optimized IBL aluminum compositions and acceptor (Mg) p-doping levels.

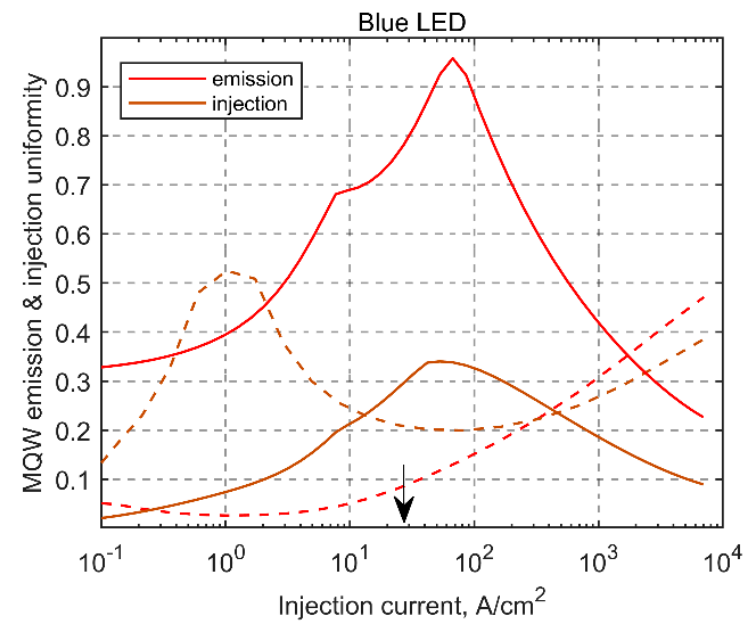

(a)

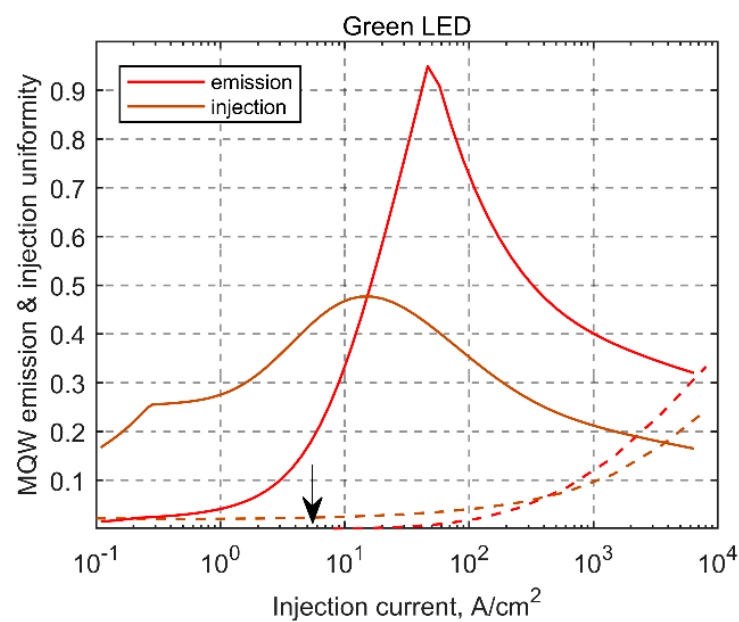

(b)

Figure 4. Injection dependence of MQW active region emission and injection uniformity characteristics in (a) blue-emitting and (b) green-emitting LEDs with plain-barrier (dashed lines) and optimized IBL (solid lines) active region design. Arrows indicate the LED injection levels corresponding to maximum external quantum efficiency (EQE). 
By combining both compositional and doping degrees of freedom, the IBL concept represents a versatile design element. Most natural IBL design applications, however, include structures which essentially require the injection distribution control over MQW active regions with only few QWs, such as monolithic tunable multi-color LEDs. Color control ability of IBL the active region has been demonstrated in the full red-green-blue (RGB) spectrum [11] and recently also used in a white-color LED with a tunable color temperature [13]. Figure 5 presents an example of chromaticity characteristics' control in the three-color IBL LED of the current design. Single-color quantum wells have been replaced here with blue, green, and red-emitting QWs, and IBLs have been re-optimized to provide white color emission at a nominal injection of $50 \mathrm{~A} / \mathrm{cm}^{2}$; see Table 2 . Figure 5 a shows the chromaticity diagram of the white-color LED with a solid black locus line spanning the LED injection range, from $0.1 \mathrm{~A} / \mathrm{cm}^{2}$ to $5 \mathrm{kA} / \mathrm{cm}^{2}$. Figure $5 \mathrm{~b}$ illustrates the evolution of the representative point on the diagram locus line; i.e., the injection dependence of the corresponding locus point chromaticity coordinates $\mathrm{x}, \mathrm{y}$, and $\mathrm{z}=1$ $-x-y$. The circles in both subplots indicate the regions of white light LED emission. To illustrate the simulation sensitivity to IBL doping and composition, Figure 5a has been completed with two additional locus lines for LEDs with $(i)$ the plain-barrier active region of the same doping profile (dashed locus line) and (ii) the active region with undoped IBLs of the same Al content (dash-dotted locus line). Both examples clearly indicate the loss of white emission if either part of optimization is skipped. Thin dashed lines in Figure $5 \mathrm{~b}$ show the injection dependence of chromaticity coordinates for an LED with an undoped plain-barrier active region.

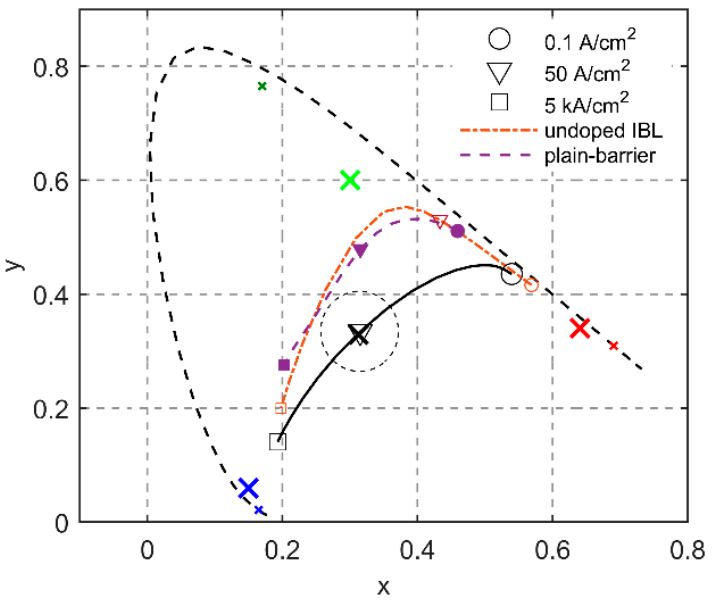

(a)

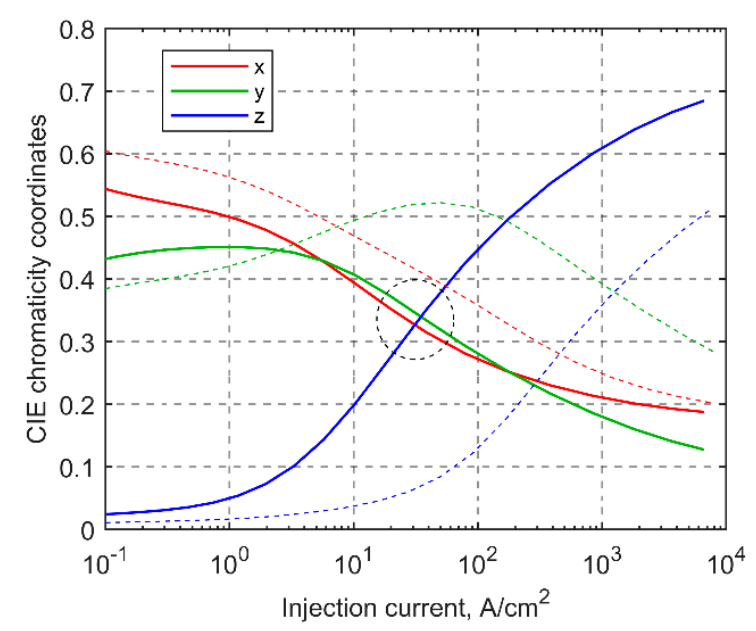

(b)

Figure 5. Injection dependence of white-color LED chromaticity characteristics. (a) chromaticity diagram of LED emission. Large cross markers indicate white light (black cross) and RGB main colors; small colored cross markers show nominal QW emission locus points. (b) Injection dependence of the chromaticity coordinates of representative points on the LED chromaticity locus line.

Table 2. IBL characteristics in simulated structures.

\begin{tabular}{cccc}
\hline LED & Aluminum, $\%$ & Acceptors, $\mathbf{1 0}^{\mathbf{1 7}} \mathbf{c m}^{-\mathbf{3}}$ & $\mathbf{P}_{\mathbf{m i n}} / \mathbf{P}_{\mathbf{m a x}}$ \\
\hline Blue, plain-barrier & - & - & 0.114 \\
Blue, IBL1/IBL2 & $23 / 12$ & $3 / 61$ & 0.910 \\
Green, plain-barrier & - & - & 0.008 \\
Green, IBL1/IBL2 & $20 / 20$ & $26 / 38$ & 0.867 \\
White, IBL1/IBL2 & $10 / 6$ & $10 / 8$ & - \\
\hline
\end{tabular}

It is worth mentioning that both our case studies employ the physics-based model simulations, with only typical material parameters, which renders the simulation results qualitative by nature. 
This raises the question about the critical parameters which can affect the presented values of optimized IBL characteristics. The question cannot be unambiguously answered at this point. Since IBL design combines the composition and doping variation, the deviations in band offsets, polarization charges and layer widths would naturally affect the results. We emphasize, however, that QW parameters should enter that list on equal footing. The IBL effect on QW injection can be different for all active QWs, depending on the balance between the carrier capture and intra-QW recombination rates, with capture rates for each type of carrier strongly affected by the adjacent IBLs. These considerations, however, require more extensive study and are beyond the means of this Communication which is intended only to illustrate the versatility of the IBL concept, and does not provide an ultimate design or final solution to the problem of MQW injection.

\section{Conclusions}

In conclusion, intermediate carrier blocking layers (IBLs) incorporated into the multi-quantum-well active region of III-nitride light-emitting structure represent a versatile active region design element, combining both compositional and doping degrees of freedom. IBLs with optimal design allow efficient control over the carrier injection distribution across the LED active region to match the application-specific injection characteristics. This approach has been exemplified in this work by MQW IBL LED design with three identical quantum wells, blue or green-emitting, uniformly populated at LED operational currents. We also illustrate the IBL design flexibility by demonstrating the possibility of white-color emission from similar designed three-QW IBL active region comprised of blue, green, and red-emitting QWs.

\section{Patents}

US Patent Application \#15173517 filed 6/6/2016.

Author Contributions: Conceptualization and supervision, H.S.E.-G.; software and formal analysis, M.V.K.; validation and project administration, C.-L.C.

Funding: This research received no external funding.

Acknowledgments: In-house software development by Denis V. Mamedov is gratefully acknowledged.

Conflicts of Interest: The authors declare no conflict of interest.

\section{References}

1. Tessler, N.; Eisenstein, G. On carrier injection and gain dynamics in quantum well lasers. IEEE J. Quantum Electron. 1993, 29, 1586-1595. [CrossRef]

2. Marcinkevičius, S.; Yapparov, R.; Kuritzky, L.Y.; Wu, Y.R.; Nakamura, S.; DenBaars, S.P.; Speck, J.S. Interwell carrier transport in InGaN/(In)GaN multiple quantum wells. Appl. Phys. Lett. 2019, 114, 151103. [CrossRef]

3. Kisin, M.V.; El-Ghoroury, H.S. Inhomogeneous injection in III-Nitride light emitters with deep multiple quantum wells. J. Comput. Electron. 2015, 14, 432-443. [CrossRef]

4. Dutta, N.K. Current injection in multiquantum well lasers. IEEE J. Quantum Electron. 1983, 19, $794-797$. [CrossRef]

5. Kisin, M.V.; El-Ghoroury, H.S. Modeling of III-Nitride multiple quantum well light emitting structures. IEEE J. Sel. Top. Quantum Electron. 2013, 19, 1901410-1-10. [CrossRef]

6. Kisin, M.V.; Chuang, C.L.; El-Ghoroury, H.S. Non-equilibrium QW populations and active region inhomogeneity in polar and nonpolar III-nitride light emitters. J. Appl. Phys. 2012, 111, 103113. [CrossRef]

7. Kisin, M.V.; El-Ghoroury, H.S. Injection characteristics of polar and nonpolar multiple-QW structures and active region ballistic overshoot. Phys. Status Solidi C 2011, 8, 2264-2266. [CrossRef]

8. Kisin, M.V.; El-Ghoroury, H.S. Modeling of color-coded III-nitride LED structures with deep quantum wells. Opt. Quantum Electron. 2014, 46, 1209-1215. [CrossRef] 
9. David, A.; Grundmann, M.J.; Kaeding, J.F.; Gardner, N.F.; Mihopoulos, T.G.; Krames, M.R. Carrier distribution in (0001) InGaN/GaN multiple quantum well light-emitting diodes. Appl. Phys. Lett. 2008, 92, 53502. [CrossRef]

10. El-Ghoroury, H.S.; Kisin, M.V.; Chuang, C.-L.; Chen, J.-C.; Yeh, Y.-C.M. Light Emitting Structures with Selective Carrier Injection into Multiple Active Layers. U.S. Patent 20160359300A1, 8 December 2016.

11. El-Ghoroury, H.S.; Yeh, M.; Chen, J.C.; Li, X.; Chuang, C.-L. Growth of monolithic full-color GaN-based LED with intermediate carrier blocking layers. AIP Adv. 2016, 6, 75316. [CrossRef]

12. Kisin, M.V.; Mamedov, D.V.; El-Ghoroury, H.S. Simulation of full-color III-nitride LED with intermediate carrier blocking layers. Opt. Quantum Electron. 2016, 48, 525. [CrossRef]

13. El-Ghoroury, H.S.; Nakajima, Y.; Yeh, M.; Liang, E.; Chung, C.-L.; Chen, J.C. Color temperature tunable white light based on monolithic color-tunable light emitting diodes. Opt. Express. 2019. under review for publication.

14. Schubert, M.F.; Schubert, E.F. Effect of heterointerface polarization charges and well width upon capture and dwell time for electrons and holes above GaInN/GaN quantum wells. Appl. Phys. Lett. 2010, 96, 131102. [CrossRef]

15. Vurgaftman, I.; Meyer, J.R. Electron band structure parameters. In Nitride Semiconductor Devices: Principles and Simulation; Piprek, J., Ed.; Wiley-VCH Verlag GmbH: Weinheim, Germany, 2007; pp. 13-48.

16. Piprek, J.; Simon Li, Z.M. Sensitivity analysis of electron leakage in III-nitride light-emitting diodes. Appl. Phys. Lett. 2013, 102, 131103. [CrossRef]

17. Schiavon, D.; Binder, M.; Peter, M.; Galler, B.; Drechsel, P.; Scholz, F. Wavelength-dependent determination of the recombination rate coefficients in single-quantum-well GaInN/GaN light emitting diodes. Phys. Status Solidi 2013, 250, 283-290. [CrossRef]

18. Mukai, T.; Yamada, M.; Nakamura, S. Characteristics of InGaN-based UV/blue/green/amber/red light-emitting diodes. Jpn. J. Appl. Phys. 1999, 38, 3976-3981. [CrossRef]

19. Karpov, S.Y.; Makarov, Y.N. Dislocation effect on light emission efficiency in gallium nitride. Appl. Phys. Lett. 2002, 81, 4721-4723. [CrossRef]

20. Di Carlo, A.; della Sala, F.; Lugli, P.; Fiorentini, V.; Bernardini, F. Doping screening of polarization fields in nitride heterostructures. Appl. Phys. Lett. 2000, 76, 3950-3952. [CrossRef]

21. Der Maur, M.A.; Pecchia, A.; Penazzi, G.; Rodrigues, W.; Di Carlo, A. Efficiency drop in green InGaN/GaN light emitting diodes: The role of random alloy fluctuations. Phys. Rev. Lett. 2016, 116, 27401. [CrossRef]

22. Polkovnikov, A.S.; Zegrya, G.G. Auger recombination in semiconductor quantum wells. Phys. Rev. B 1998, 58, 4039-4056. [CrossRef]

23. Iveland, J.; Martinelli, L.; Peretti, J.; Speck, J.S.; Weisbuch, C. Direct measurement of auger electrons Emitted from a semiconductor light-emitting diode under electrical injection: Identification of the dominant mechanism for efficiency droop. Phys. Rev. Lett. 2013, 110, 177406. [CrossRef] [PubMed]

24. Binder, M.; Nirschl, A.; Zeisel, R.; Hager, T.; Lugauer, H.J.; Sabathil, M.; Bougeard, D.; Wagner, J.; Galler, B. Identification of nnp and npp Auger recombination as significant contributor to the efficiency droop in (GaIn)N quantum wells by visualization of hot carriers in photoluminescence. Appl. Phys. Lett. 2013, 103, 71108. [CrossRef]

25. Bulashevich, K.A.; Karpov, S.Y. Is Auger recombination responsible for the efficiency rollover in III-nitride light-emitting diodes? Phys. Status Solidi C 2008, 5, 2066-2069. [CrossRef]

26. Piprek, J. Efficiency droop in nitride-based light-emitting diodes. Phys. Status Solidi A 2010, 207, $2217-2225$. [CrossRef]

27. Shim, J.-I. Internal quantum efficiency. In III-Nitride Based Light Emitting Diodes and Applications; Seong, T.-Y., Han, J., Amano, H., Morkoç, H., Eds.; Springer: Singapore, 2017; pp. 163-207.

28. Luryi, S. An induced base hot-electron transistor. IEEE Electron. Device Lett. 1985, 6, 178-180. [CrossRef]

(C) 2019 by the authors. Licensee MDPI, Basel, Switzerland. This article is an open access article distributed under the terms and conditions of the Creative Commons Attribution (CC BY) license (http://creativecommons.org/licenses/by/4.0/). 